\title{
Shape-Dependent Energy of an Elliptical Jellium Background
}

\author{
Orion Ciftja, LeDarion Escamilla, and Ryan Mills \\ Department of Physics, Prairie View A\&M University, Prairie View, TX 77446, USA \\ Correspondence should be addressed to Orion Ciftja; ogciftja@pvamu.edu
}

Received 7 April 2015; Accepted 27 April 2015

Academic Editor: Sergei Sergeenkov

Copyright (C) 2015 Orion Ciftja et al. This is an open access article distributed under the Creative Commons Attribution License, which permits unrestricted use, distribution, and reproduction in any medium, provided the original work is properly cited.

\begin{abstract}
The jellium model is commonly used in condensed matter physics to study the properties of a two-dimensional electron gas system. Within this approximation, one assumes that electrons move in the presence of a neutralizing background consisting of uniformly spread positive charge. When properties of bulk systems (of infinite size) are studied, shape of the jellium domain is irrelevant. However, the same cannot be said when one is dealing with finite systems of electrons confined in a finite two-dimensional region of space. In such a case, geometry and shape of the jellium background play a role on the overall properties of the system. In this work, we assume that the region where the electrons are confined is represented by a jellium background charge with an elliptical shape. It is shown that, in this case, the Coulomb self-energy of the elliptically shaped region can be exactly calculated in closed analytical form by using suitable mathematical transformations. The results obtained reveal the external influence of geometry/shape on the properties of two-dimensional systems of few electrons confined to a small finite region of space.
\end{abstract}

The two-dimensional electron gas (2DEG) model has received a great deal of attention in condensed matter physics due to the richness and complexity of the emerging phenomena associated with it. The variety of possible scenarios makes this model fascinating from a theoretical and experimental point of view. In particular, a 2DEG in a strong perpendicular magnetic field has come to the forefront of current research as a result of the discovery of the integer quantum Hall effect (IQHE) $[1,2]$ and the fractional quantum Hall effect (FQHE) [3-7] phenomena. Any theoretical model for a 2DEG is required to guarantee the overall charge neutrality of the system. The simplest way to satisfy this condition is to assume that electrons move in presence of a charge neutralizing background. A common approach is to adopt the so-called "jellium" approximation. Within this approximation, one assumes that the neutralizing positive charge is uniformly spread in a two-dimensional (2D) region that contains the electrons. The whole system of $N$ electrons in presence of a uniformly charged positive background is, thus, seen as a 2 DEG system in the jellium approximation. One typically assumes a uniform finite density for the system, $\rho_{0}=N / A$, where $N$ is the number of electrons and $A$ is the area of the 2D system. In the thermodynamic limit, both $N \rightarrow \infty$ and $A \rightarrow \infty$, though their ratio is finite. The 2DEG jellium model is widely studied in condensed matter physics with the main interest in the thermodynamic limit [8-10]. Based on the above consideration, one immediately concludes that the shape chosen for the jellium background is irrelevant when it comes to studies of properties of the system in the thermodynamic limit.

However, there have been recent developments in the field of nanotechnology that make possible the fabrication of finite systems of few electrons confined in a finite 2D domain [1116]. Typical experimental techniques involve placement of patterned gate electrodes, or etching on a bulk 2DEG system so that a small finite area of the sample is depleted of almost all electrons except for the few ones targeted for confinement. This domain depleted of electrons can be modeled as a positively uniformly charged finite background. Since now this domain is finite, its shape that is generally determined from the given experimental setup matters. Hence, studies of finite system of electrons are sensitive to the geometry of the background in contrast to the treatment of their counterparts in the thermodynamic limit. As a result, the properties of a finite $2 \mathrm{DEG}$ reflect the shape chosen for the positive background domain $[17,18]$ for the case under consideration. For example, in a typical semiconductor quantum dot system of few electrons, one typically assumes circular 
symmetry for the confinement region. However, many times the experimental setup is such that the confinement region where the electrons are trapped is noncircular. In such a case, the shape of such domain will affect the properties of a finite system. In this work, we assume that the area occupied by electrons is noncircular and has an elliptical shape. The objective is to calculate exactly the Coulomb self-energy of a uniformly charged jellium background with elliptical shape. This calculation is a necessary step in order to calculate accurately the total energy of a finite 2DEG confined to a domain with elliptical shape. Analytical results derived can be used in systematic studies of the properties of finite $2 \mathrm{DEG}$ of $N$ electrons embedded in a neutralizing background with elliptical shape.

We consider a uniformly charged 2D domain with elliptical shape (elliptical plate) with area $\pi a b$, where $a$ is the semimajor axis, $b$ is the semiminor axis, and $a \geq b>0$. This region contains a uniformly distributed positive charge, $Q$. Thus, the uniform surface charge density is given as

$$
\rho_{0}=\frac{Q}{\pi a b} \text {. }
$$

We assume Coulomb interaction between elementary charges $d q_{1}=\rho_{0} d^{2} r_{1}$ and $d q_{2}=\rho_{0} d^{2} r_{2}$ and write the expression for the self-energy of the elliptical domain as

$$
E_{s}=\frac{k_{e} \rho_{0}^{2}}{2} \int_{D} d^{2} r_{1} \int_{D} d^{2} r_{2} \frac{1}{\left|\vec{r}_{1}-\vec{r}_{2}\right|},
$$

where $k_{e}$ is Coulomb's electric constant, $\vec{r}_{i}=\left(x_{i}, y_{i}\right)(i=1,2)$ are $2 \mathrm{D}$ position vectors, and $D$ is the elliptical domain of integration:

$$
D: \frac{x^{2}}{a^{2}}+\frac{y^{2}}{b^{2}} \leq 1
$$

Note that the calculation in (2) involves a nontrivial fourdimensional integral over a noncircular (elliptical) domain. The calculation of the quantity in (2) is facilitated if we use the general 2D Fourier transform formula:

$$
\frac{1}{\left|\vec{r}_{1}-\vec{r}_{2}\right|}=\int \frac{d^{2} q}{(2 \pi)^{2}} e^{-i \vec{q}\left(\vec{r}_{1}-\vec{r}_{2}\right)} \frac{2 \pi}{q} .
$$

Substitution of the expression from (4) into (2) gives

$$
E_{s}=\frac{k_{e} \rho_{0}^{2}}{2} \int \frac{d^{2} q}{(2 \pi)^{2}} \frac{2 \pi}{q} \int_{D} d^{2} r_{1} e^{-i \vec{q} \vec{r}_{1}} \int_{D} d^{2} r_{2} e^{+i \vec{q} \vec{r}_{2}}
$$

We see now that the full solution of the problem involves the calculation of the following integral:

$$
I=\int_{D} d^{2} r e^{i \vec{q} \vec{r}}=\iint_{D} d x d y e^{i\left(q_{x} x+q_{y} y\right)}
$$

In order to calculate the above integral, we introduce two new variables:

$$
\begin{aligned}
& u=\frac{x}{a} \\
& v=\frac{y}{b} .
\end{aligned}
$$

For this set of new variables, the ellipse appears as a unit circle:

$$
D_{c}: u^{2}+v^{2} \leq 1
$$

In terms of new variables,

$$
I=(a b) \iint_{D_{c}} d u d v e^{i\left(a q_{x} u+b q_{y} v\right)},
$$

where the domain of integration, $D_{c}$, is a unit circle. In order to simplify the notation, let us define two new vectors, $\vec{Q}=$ $\left(Q_{x}, Q_{y}\right)$ and $\vec{R}=\left(R_{x}, R_{y}\right)$ which have the following vector components:

$$
\begin{aligned}
& \vec{Q}=\left(a q_{x}, b q_{y}\right) ; \\
& \vec{R}=(u, v) .
\end{aligned}
$$

With this notation, the expression in (9) can be written as

$$
\begin{aligned}
I & =(a b) \int_{D_{c}:|\vec{R}| \leq 1} d^{2} R e^{i \vec{Q} \vec{R}} \\
& =(a b) \int_{0}^{1} d R R \int_{0}^{2 \pi} d \theta e^{i Q R \cos (\theta)},
\end{aligned}
$$

where $\theta$ is the angle between $\vec{R}$ and $\vec{Q}$ and

$$
Q=\sqrt{Q_{x}^{2}+Q_{y}^{2}}=\sqrt{a^{2} q_{x}^{2}+b^{2} q_{y}^{2}} .
$$

The angular integration gives a term that involves the 0th order Bessel function; thus,

$$
I=2 \pi(a b) \int_{0}^{1} d R R J_{0}(Q R)=2 \pi(a b) \frac{J_{1}(Q)}{Q},
$$

where we used the well-known formula:

$$
\int d x x J_{0}(x)=x J_{1}(x) .
$$

At this point, we proved that two of the integrals appearing in (5) have the form

$$
\int_{D} d^{2} r_{1} e^{-i \vec{q} \vec{r}_{1}}=\int_{D} d^{2} r_{2} e^{+i \vec{q} \vec{r}_{2}}=2 \pi(a b) \frac{J_{1}(Q)}{Q},
$$

where $\vec{Q}$ is related to $\vec{q}$ through the relations specified in (10). Thus, the expression for self-energy in (5) can now be written as

$$
E_{s}=\frac{k_{e} \rho_{0}^{2}}{2} \int \frac{d^{2} q}{(2 \pi)^{2}} \frac{2 \pi}{q}\left[2 \pi(a b) \frac{J_{1}(Q)}{Q}\right]^{2} .
$$

We recall that the domain of integration of $\vec{q}$ includes the whole $\vec{q}$-space. To simplify the integration in (16), we change to polar coordinates $\left(q, \theta_{q}\right)$ and write

$$
\begin{aligned}
& q_{x}=q \cos \left(\theta_{q}\right) ; \\
& q_{y}=q \sin \left(\theta_{q}\right) .
\end{aligned}
$$


In terms of $q$ and $\theta_{q}$, the variable $Q$ is

$$
Q=q \sqrt{a^{2} \cos ^{2}\left(\theta_{q}\right)+b^{2} \sin ^{2}\left(\theta_{q}\right)} .
$$

After rewriting (16) in polar coordinates, we obtain

$$
\begin{aligned}
E_{s}= & \frac{k_{e} \rho_{0}^{2}}{2}(a b)^{2} 2 \pi \int_{0}^{\infty} d q \int_{0}^{2 \pi} d \theta_{q} \\
& \cdot\left[\frac{J_{1}\left(q \sqrt{a^{2} \cos ^{2}\left(\theta_{q}\right)+b^{2} \sin ^{2}\left(\theta_{q}\right)}\right)}{q \sqrt{a^{2} \cos ^{2}\left(\theta_{q}\right)+b^{2} \sin ^{2}\left(\theta_{q}\right)}}\right]^{2} .
\end{aligned}
$$

Since the order of integration is irrelevant, we choose to perform, first, the integration relative to the variable, $q$, and then the angular integration. For simplicity, let us denote

$$
\alpha\left(\theta_{q}\right)=\sqrt{a^{2} \cos ^{2}\left(\theta_{q}\right)+b^{2} \sin ^{2}\left(\theta_{q}\right)},
$$

where $\alpha\left(\theta_{q}\right)$ is a term that depends only on the angular variable, but not $q$. It turns out the integration over the variable $q$ is rather standard and results in

$$
\int_{0}^{\infty} d q\left\{\frac{J_{1}\left[\alpha\left(\theta_{q}\right) q\right]}{\alpha\left(\theta_{q}\right) q}\right\}^{2}=\frac{4}{3 \pi} \frac{1}{\alpha\left(\theta_{q}\right)} .
$$

Based on this result, (19) becomes

$$
\begin{aligned}
& E_{s} \\
& =\frac{k_{e} \rho_{0}^{2}}{2}(a b)^{2} \frac{8}{3} \int_{0}^{2 \pi} d \theta_{q} \frac{1}{\sqrt{a^{2} \cos ^{2}\left(\theta_{q}\right)+b^{2} \sin ^{2}\left(\theta_{q}\right)}} .
\end{aligned}
$$

The denominator of the integrand in the expression above can be written as

$$
\sqrt{a^{2} \cos ^{2}\left(\theta_{q}\right)+b^{2} \sin ^{2}\left(\theta_{q}\right)}=a \sqrt{1-m \sin ^{2}\left(\theta_{q}\right)}
$$

where $m$ represents the following shape-dependent parameter:

$$
m=\frac{\left(a^{2}-b^{2}\right)}{a^{2}} .
$$

Note that $m$ satisfies the condition $0 \leq m<1$ since $a \geq b>0$. Simple integral formulas involving trigonometric functions allow us to rewrite (22) as

$$
E_{s}=\frac{16}{3} k_{e} \rho_{0}^{2}(a b)^{2} \frac{1}{a} \int_{0}^{\pi / 2} d \theta_{q} \frac{1}{\sqrt{1-m \sin ^{2}\left(\theta_{q}\right)}} .
$$

Thus, the final expression for the self-energy becomes

$$
E_{s}=\frac{16}{3} k_{e} \rho_{0}^{2}(a b)^{2} \frac{1}{a} K(m),
$$

where

$$
K(m)=\int_{0}^{\pi / 2} d \theta \frac{1}{\sqrt{1-m \sin ^{2}(\theta)}}, \quad 0 \leq m<1,
$$

represents the complete elliptical integral of the first kind [19, 20]. Special limiting values of this function are $K(m=0)=$ $\pi / 2$ and $K(m \rightarrow 1) \rightarrow \infty$.

At this juncture, we write the expression in (26) as

$$
E_{s}=\frac{16}{3} k_{e} \rho_{0}^{2}(a b)^{2} \frac{1}{a} K\left[\frac{a^{2}-b^{2}}{a^{2}}\right] .
$$

This way we can compare our result above to an earlier expression (denoted as E) reported by Keller et al. [21] obtained through a different direct integration method:

$$
E=\frac{16}{3} k_{e} \rho_{0}^{2}(a b)^{2} \frac{1}{b} K\left[\frac{b^{2}-a^{2}}{a^{2}}\right] .
$$

The following formula for complete elliptic integrals of the first kind (with $a, b$ any two numbers) applies

$$
\frac{1}{a} K\left[\frac{a^{2}-b^{2}}{a^{2}}\right]=\frac{1}{b} K\left[\frac{b^{2}-a^{2}}{b^{2}}\right] .
$$

With this transformation, our result in (28) can be rewritten as

$$
E_{s}=\frac{16}{3} k_{e} \rho_{0}^{2}(a b)^{2} \frac{1}{b} K\left[\frac{b^{2}-a^{2}}{b^{2}}\right] .
$$

A comparison of (31) to (29) seems to suggest that that the previous result reported in [21], namely, (29), contains a possible typo and should be corrected as in (31) [by replacing " $a$ " with " $b^{2}$ " in the denominator of the argument of the function $K$ appearing in (29)].

A uniformly charged ellipse turns into a uniformly charged disk with radius $R$ for the special case of $a=$ $b=R$. As a result, (26) reduces to the known result of the electrostatic self-energy of a uniformly charged disk [22] that reads

$$
E_{\text {disk }}=\frac{8 \pi}{3} k_{e} \rho_{0}^{2} R^{3}=\frac{8}{3 \pi} \frac{k_{e} Q^{2}}{R},
$$

where $Q$ is the total charge, $R$ is the radius of the disk, and $k_{e}$ is Coulomb's electric constant.

To conclude, in this work we focused our attention on a finite $2 \mathrm{D}$ jellium model with an elliptical shape. We show that in such a case the Coulomb self-energy of the positive background can be analytically calculated in closed form by using simple mathematical transformations. The results derived can be used in systematic studies of the properties of finite systems of electrons embedded in a elliptically shaped background of uniform positive charge. One can also use the above formulas to derive exact expressions for systems of few $N$ electrons in an elliptical geometry and compare such results to corresponding systems of few electrons in a disk geometry [23-25] or other geometries. This way, one can study the influence of the shape of the confinement domain on the overall properties of the finite $2 \mathrm{D}$ system. Studies of a finite 2DEG of this nature may reveal subtle effects arising from the influence of a noncircular shape on the overall properties of the system of few embedded electrons in a uniformly charged background. 


\section{Conflict of Interests}

The authors declare that there is no conflict of interests regarding the publication of this paper.

\section{Acknowledgments}

This research was supported in part by US Army Research Office (ARO) Grant no. W911NF-13-1-0139 and National Science Foundation (NSF) Grants nos. DMR-1104795 and DMR-1410350.

\section{References}

[1] K. vo Klitzing, G. Dorda, and M. Pepper, "New method for highaccuracy determination of the fine-structure constant based on quantized hall resistance," Physical Review Letters, vol. 45, article 494, 1980 .

[2] O. Ciftja, "Exact results for finite quantum Hall systems of electrons at filling factor one: disk geometry," Journal of Mathematical Physics, vol. 52, no. 12, Article ID 122105, 2011.

[3] D. C. Tsui, H. L. Stormer, and A. C. Gossard, "Two-dimensional magnetotransport in the extreme quantum limit," Physical Review Letters, vol. 48, no. 22, pp. 1559-1562, 1982.

[4] R. B. Laughlin, "Anomalous quantum hall effect: an incompressible quantum fluid with fractionally charged excitations," Physical Review Letters, vol. 50, no. 18, pp. 1395-1398, 1983.

[5] J. K. Jain, "Composite-fermion approach for the fractional quantum Hall effect," Physical Review Letters, vol. 63, pp. 199$202,1989$.

[6] O. Ciftja, "Theoretical estimates for the correlation energy of the unprojected composite fermion wave function," Physica E: LowDimensional Systems and Nanostructures, vol. 9, no. 2, pp. 226230, 2001

[7] F. D. Haldane, "Fractional quantization of the Hall effect: a hierarchy of incompressible quantum fluid states," Physical Review Letters, vol. 51, article 605, 1983.

[8] D. Ceperley, "Ground state of the fermion one-component plasma: a Monte Carlo study in two and three dimensions," Physical Review B, vol. 18, no. 7, pp. 3126-3138, 1978.

[9] B. Bernu, F. Delyon, M. Holzmann, and L. Baguet, "HartreeFock phase diagram of the two-dimensional electron gas," Physical Review B, vol. 84, no. 11, Article ID 115115, 2011.

[10] B. Bernu, F. Delyon, M. Duneau, and M. Holzmann, "Metalinsulator transition in the Hartree-Fock phase diagram of the fully polarized homogeneous electron gas in two dimensions," Physical Review B, vol. 78, no. 24, Article ID 245110, 2008.

[11] O. Ciftja, "Classical behavior of few-electron parabolic quantum dots," Physica B: Condensed Matter, vol. 404, no. 12-13, pp. 16291631, 2009.

[12] M. B. Tavernier, E. Anisimovas, F. M. Peeters, B. Szafran, J. Adamowski, and S. Bednarek, "Four-electron quantum dot in a magnetic field," Physical Review B, vol. 68, Article ID 205305, 2003.

[13] O. Ciftja and M. G. Faruk, "Two-dimensional quantum-dot helium in a magnetic field: variational theory," Physical Review $B$, vol. 72, Article ID 205334, 2005.

[14] O. Steffens, U. Rossler, and M. Suhrke, "Generalized Hund's rule in the addition spectrum of a quantum dot," Europhysics Letters, vol. 42 , no. 5 , p. 529, 1998.
[15] J. Kainz, S. A. Mikhailov, A. Wensauer, and U. Rössler, "Quantum dots in high magnetic fields: calculation of ground-state properties," Physical Review B, vol. 65, no. 11, Article ID 115305, 2002.

[16] U. Merkt, J. Huser, and M. Wagner, "Energy spectra of two electrons in a harmonic quantum dot," Physical Review B, vol. 43, no. 9, pp. 7320-7323, 1991.

[17] O. Ciftja, B. Sutton, and A. Way, "Energy in a finite twodimensional spinless electron gas," AIP Advances, vol. 3, no. 5, Article ID 052110, 2013.

[18] O. Ciftja, "Hartree-Fock energy of a finite two-dimensional electron gas system in a jellium background," Physica B, vol. 458, pp. 92-97, 2015.

[19] M. Abramowitz and I. A. Stegun, Handbook of Mathematical Functions, Dover, New York, NY, USA, 9th edition, 1970.

[20] G. B. Arfken and H. J. Weber, Mathematical Methods for Physicists, Academic Press, San Diego, Calif, USA, 4th edition, 1995.

[21] D. J. Keller, J. P. Korb, and H. M. McConnell, "Theory of shape transitions in two-dimensional phospholipid domains," Journal of Physical Chemistry, vol. 91, no. 25, pp. 6417-6422, 1987.

[22] O. Ciftja, "Monte Carlo study of Bose Laughlin wave function for filling factors 1/2,1/4 and 1/6," Europhysics Letters, vol. 74, no. 3, pp. 486-492, 2006.

[23] O. Ciftja, "Exact results for systems of electrons in the fractional quantum Hall regime," Physica B: Condensed Matter, vol. 404, no. 2, pp. 227-230, 2009.

[24] O. Ciftja, "Exact results for systems of electrons in the fractional quantum Hall regime II," Physica B: Condensed Matter, vol. 404, no. 16, pp. 2244-2246, 2009.

[25] R. Morf and B. I. Halperin, "Monte Carlo evaluation of trial wave functions for the fractional quantized Hall effect: disk geometry," Physical Review B, vol. 33, no. 4, pp. 2221-2246, 1986. 

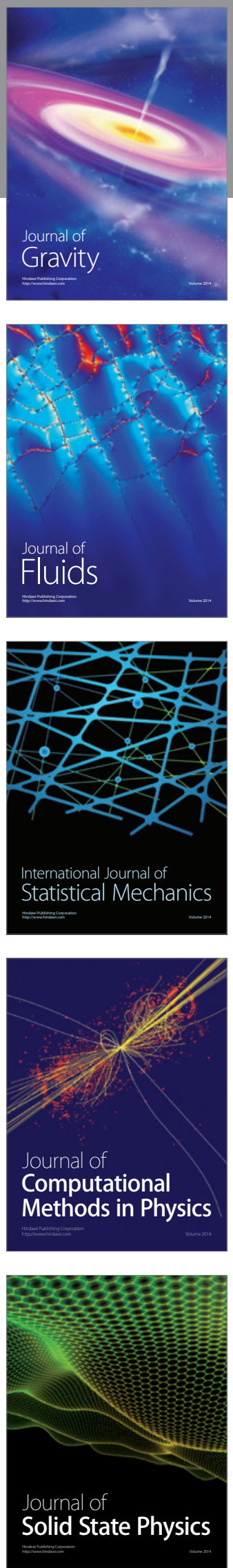

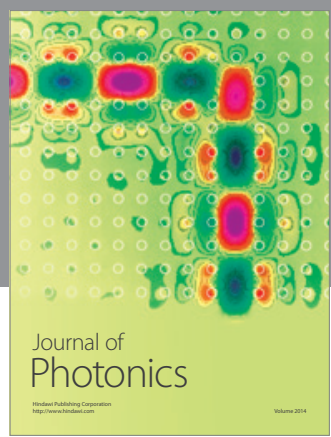

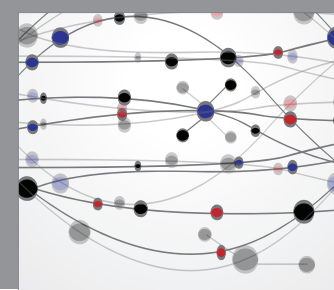

The Scientific World Journal

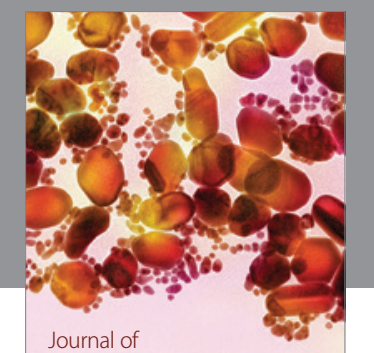

Soft Matter
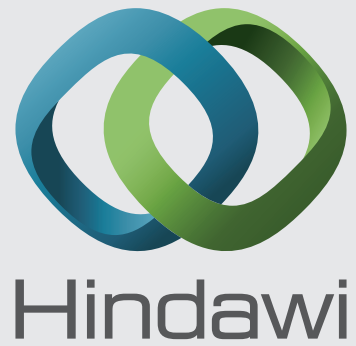

Submit your manuscripts at

http://www.hindawi.com
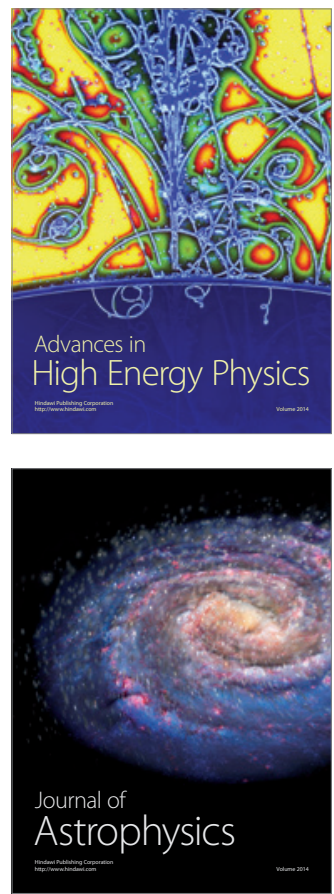
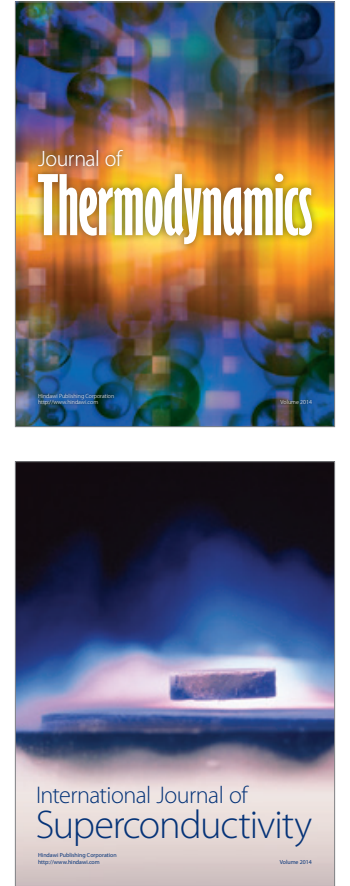
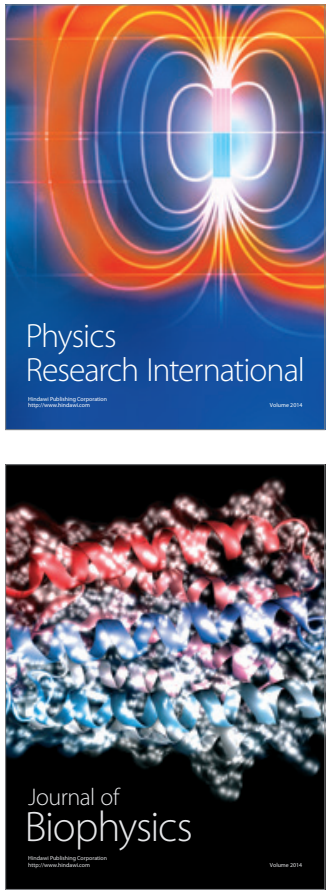
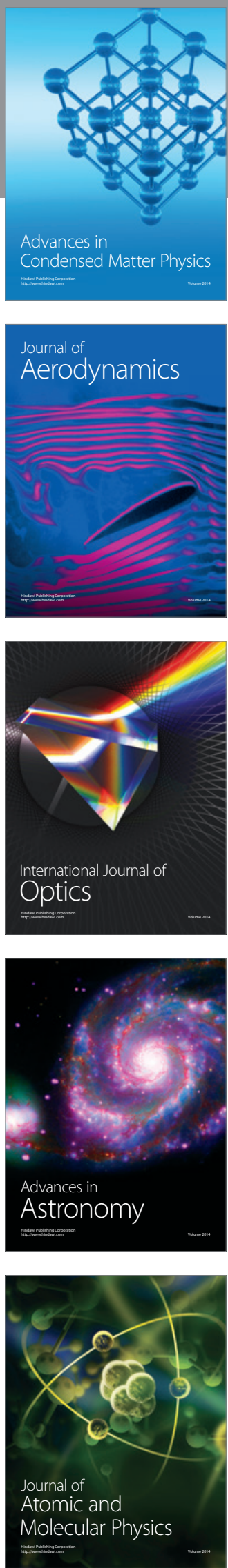